\title{
Le chômage en Europe
}

Qu'avons-nous appris?

Unemployment in UE : what did we learn about it?

\section{Daniel Cohen}

\section{(2) OpenEdition \\ Journals}

Édition électronique

URL : http://journals.openedition.org/travailemploi/3596

DOI : $10.4000 /$ travailemploi.3596

ISSN : 1775-416X

Éditeur

DARES - Ministère du Travail

Édition imprimée

Date de publication : 5 juin 2009

Pagination : 49-60

ISSN : 0224-4365

\section{Référence électronique}

Daniel Cohen, « Le chômage en Europe », Travail et Emploi [En ligne], 118 | avril-juin 2009, mis en ligne le 03 décembre 2010, consulté le 30 avril 2019. URL : http://journals.openedition.org/

travailemploi/3596; DOI : 10.4000/travailemploi.3596 


\title{
Le chômage en Europe: qu'avons-nous appr is ?
}

\author{
Daniel Cohen $(*)$
}

Beaucoup de transformations se sont opérées depuis la publication du célèbre Job Study de l'OCDE, il y a plus d'une dizaine d'années. L'environnement macroéconomique tout d'abord n'a plus rien à voir avec celui de 1994. À l'époque, les pays industrialisés sortaient tout juste d'une période de grande inflation, et les instruments d'analyse du chômage en portaient la marque. Aujourd'hui, l'inflation a quasiment disparu. Cela ne veut pas dire qu'il n'y ait plus de danger mais qu'une remise à plat des notions héritées de cette période s'impose. Des concepts tels le NAIRU ont-ils encore un sens, quand l'inflation est si basse? laquelle le marché du travail pouvait parvenir à l'équilibre de multiples manières.

Comme les données rassemblées au tableau ci-dessous le montrent, le chômage a connu une constellation de trajectoire au cours des dernières décennies.

Le premier constat simple qui émerge de ce tableau est le résultat bien connu suivant: les pays où le chômage était le plus bas à la fin des années 1960 sont ceux où il est le plus élevé aujourd'hui. C'est notamment le cas des trois grands pays d'Europe continentale: l'Allemagne, la France ou l'Italie

Tableau 1 : Taux de chômage (en \%)

\begin{tabular}{|l|c|c|c|c|c|c|c|}
\hline & $\mathbf{1 9 6 6 - 1 9 7 3}$ & $\mathbf{1 9 7 4 - 1 9 7 9}$ & $\mathbf{1 9 8 0 - 1 9 8 5}$ & $\mathbf{1 9 8 6 - 1 9 9 2}$ & $\mathbf{1 9 9 3 - 1 9 9 8}$ & $\mathbf{1 9 9 9 - 2 0 0 5}$ & $\mathbf{2 0 0 6}$ \\
\hline Belgique & 2,7 & 6,0 & 11,6 & 8,0 & 9,4 & 7,8 & 8,2 \\
\hline Danemark & 1,0 & 6,0 & 8,8 & 6,6 & 6,7 & 4,9 & 3,8 \\
\hline France & 2,0 & 4,3 & 8,1 & 9,3 & 11,3 & 9,3 & 9,1 \\
\hline Allemagne & 0,8 & 3,4 & 6,0 & 5,9 & 8,4 & 8,4 & 8,4 \\
\hline Pays-Bas & 1,9 & 3,8 & 10,4 & 6,6 & 5,7 & 3,4 & 3,9 \\
\hline Italie & 3,4 & 5,3 & 8,9 & 9,2 & 10,9 & 9,0 & $6,8 *$ \\
\hline Royaume-Uni & 3,4 & 5,0 & 11,5 & 8,9 & 8,1 & 5,1 & $5,5 *$ \\
\hline États-Unis & 4,5 & 6,8 & 8,1 & 6,3 & 5,6 & 5,0 & 4,6 \\
\hline Japon & 1,2 & 1,9 & 2,4 & 2,4 & 3,2 & 4,9 & 4,1 \\
\hline Autriche & 1,4 & 1,7 & 3,2 & 3,4 & 4,2 & 4,2 & 4,8 \\
\hline Finlande & 2,6 & 4,6 & 5,1 & 5,8 & 14,5 & 9,2 & 7,8 \\
\hline Norvège & 1,5 & 1,8 & 2,6 & 4,4 & 5,0 & 4,0 & 3,5 \\
\hline Suède & 2,1 & 2,0 & 3,2 & 2,7 & 9,2 & 5,7 & $4,8 *$ \\
\hline Suisse & 0,0 & 1,2 & 1,9 & 2,4 & 3,8 & 3,5 & $3,2 *$ \\
\hline
\end{tabular}

Sources: bases en ligne de l'OCDE (http://stats.oecd.org/wbos); BEAN (1994) pour les données manquantes (en italique dans le tableau).

Note: moyennes pluriannuelles des taux de chômage. Taux de chômage standardisés à partir de 1986. Pour l'Allemagne, les données font référence à la RFA jusqu'en 1991. Pour 2006, les chiffres marqués par un astérisque se réfèrent à février 2007, source nationale compilée par The Economist.

La deuxième rupture s'est opérée dans le domaine des idées. La rapidité avec laquelle la Suède et la Finlande ont retrouvé des niveaux de chômage faible, malgré la crise qui les affectées au début des années 1990, ou l'exemple réussi de la flexi-sécurité danoise par exemple, ont confirmé et généralisé l'intuition de CALmFors et Driffill (1989), selon

\footnotetext{
* PSE et CEPREMAP; daniel.cohen@ens.fr. Je remercie Francesco Avvisati pour les mises à jour contenues dans cet article.
}

dans la première moitié des années 2000. Ce constat pose un problème important aux explications du chômage par les institutions. Car s'il est vrai que le marché du travail européen offre plus de sécurité d'emploi que le marché américain, ce n'est pas un phénomène récent. Beaucoup des réglementations actuellement en vigueur en Europe existaient déjà dans les années 1960, quand le taux de chômage était bas. Beaucoup de ces «rigidités» sont en fait beaucoup moins importantes aujourd'hui qu'elles ne l'étaient il y a dix ans. 
Second constat: c'est à partir de 1993-1998, après la publication du Job Study de l'OCDE, que se creusent véritablement les différences entre ces trois pays et le reste de l'Europe. La majorité des pays européens enregistre des taux désormais proches de $5 \%$. En toute hypothèse, parler de «chômage européen» n'a plus aucun sens.

\section{Les causes supposées du chômage(1)}

Nous allons faire porter notre comparaison sur quatre pays qui illustrent la diversité de ces trajectoires : les États-Unis, la France, le Danemark et les Pays-Bas.

Prenons la liste des principales rigidités du marché du travail dans plusieurs pays européens auxquelles font ordinairement allusion les tenants du rôle des institutions :

\section{Les prélèvements obligatoires}

«Les salaires ne représentent qu'une partie du coût du travail. Il faut y ajouter les cotisations sociales employeur, les cotisations retraite, etc. ». Le tableau suivant donne les prélèvements obligatoires.

Tableau 2 : Prélèvements obligatoires (en \%)

\begin{tabular}{|l|c|c|c|}
\hline & $\mathbf{1 9 9 0 - 1 9 9 4}$ & $\mathbf{1 9 9 5 - 1 9 9 9}$ & $\mathbf{2 0 0 0 - 2 0 0 3}$ \\
\hline États-Unis & 27,2 & 28,7 & 27,7 \\
\hline France & 42,4 & 44,1 & 43,8 \\
\hline Danemark & 48,2 & 49,8 & 49,1 \\
\hline Pays-Bas & 44,8 & 41,4 & 39,7 \\
\hline
\end{tabular}

Note: recettes fiscales totales en pourcentage du PIB.

Les États-Unis, comme il est bien connu, se situent à des niveaux plus bas que les trois autres pays. Les niveaux restent relativement stables en pourcentage du PIB, à l'exception des Pays-Bas où ils ont baissé de 5 points. Ils montent d'un rythme égal en France et au Danemark.

\section{Le rôle des syndicats}

«Les syndicats sont réputés plus puissants en Europe qu'aux États-Unis, poussant les salaires à la hausse et imposant des contraintes sur l'organisation du travail dans les entreprises. Les chiffres sont les s uivants ».
Tableau 3 : Taux de syndicalisation (en \%)

\begin{tabular}{|l|c|c|c|c|c|r|}
\hline & $\mathbf{1 9 6 9 -}$ & $\mathbf{1 9 7 4 -}$ & $\mathbf{1 9 8 0 -}$ & $\mathbf{1 9 8 6 -}$ & $\mathbf{1 9 9 1 -}$ & $\mathbf{1 9 9 7 -}$ \\
& $\mathbf{1 9 7 3}$ & $\mathbf{1 9 7 9}$ & $\mathbf{1 9 8 5}$ & $\mathbf{1 9 9 0}$ & $\mathbf{1 9 9 6}$ & $\mathbf{2 0 0 1}$ \\
\hline États-Unis & 26,8 & 26,4 & 19,7 & 16,2 & 14,8 & 13,2 \\
\hline France & 21,8 & 21,1 & 16,3 & 11,3 & 10,0 & 9,7 \\
\hline Danemark & 61,6 & 72,7 & 79,5 & 75,4 & 76,8 & 75,4 \\
\hline Pays-Bas & 36,4 & 36,9 & 31,9 & 25,5 & 25,6 & 24,1 \\
\hline
\end{tabular}

Source: ensemble de données «Syndiqués et salariés» disponible sur stats.oecd.org/wbos

Note: adhérents à un syndicat en pourcentage de l'emploi salarié

On voit ici un parallélisme frappant entre la France et les États-Unis, le taux de syndicalisation étant réduit de moitié dans les deux cas. La baisse est plus modérée aux Pays-Bas. Le Danemark enregistre en revanche une hausse du taux de syndicalisation au cours de la période.

On peut relier cette évolution aux travaux de BlanCHARD et PHILIPPON (2005): la syndicalisation ou le nombre de jours de grève ont un faible pouvoir explicatif sur le chômage aujourd'hui. Le climat social des années 1960 en revanche a un meilleur pouvoir explicatif, ce qui montre que des données sous-jacentes sur la qualité des relations sociales jouent vraisemblablement un rôle plus profond que ne le révèle le nombre observé de militants syndicaux.

\section{Allocations chômage}

«Les allocations chômage, plus généreuses en Europe qu'aux États-Unis, représentent une proportion plus importante du salaire. Elles sont aussi plus faciles à obtenir, et plus faciles à prolonger. Les chômeurs seraient donc moins motivés pour chercher du travail. Les chiffres sont les suivants».

Tableau 4 : Taux de remplacement net

\begin{tabular}{|l|c|c|c|}
\hline & $\begin{array}{c}\text { Moyen, sur 60 } \\
\text { mois (1995) }\end{array}$ & $\begin{array}{c}\text { Moyen, sur 60 } \\
\text { mois (2004) }\end{array}$ & $\begin{array}{c}\text { Initial } \\
\text { (2004) }\end{array}$ \\
\hline États-Unis & 42 & 36 & 54 \\
\hline France & 53 & 57 & 75 \\
\hline Danemark & ND & 70 & 70 \\
\hline Pays-Bas & ND & 66 & 74 \\
\hline
\end{tabular}

Source: tableau 3.2 de OECD Employment Outlook, 2006.

Note: le taux de remplacement net est le rapport entre le revenu net d'un chômeur et son revenu net avant de perdre l'emploi, calculé par l'OCDE comme une moyenne entre différents cas «typiques» considérés. Le taux de remplacement net moyen sur soixante mois inclut tous les revenus de l'assistance pour lequel le chômeur est éligible.

Le même raisonnement que celui utilisé pour les syndicats s'applique ici: les indemnités chômage n'ont pas la même signification dans des environnements aussi différents que le Danemark ou la France. Tout dépend de la manière dont les institutions encadrent le comportement des chômeurs (voir sur ce point les travaux de Yann Algan et Pierre CAHuc, 2005 et 2006).
(1) Cette section reprend et actualise certains aspects de la version française du livre Macroéconomie d'Olivier Blanchard adapté en français par l'auteur (les phrases entre guillemets sont reprises du livre). Je remercie Cyril Nouveau pour son aide pour cette adaptation. 


\section{Salaire minimum et travail non qualifié}

«Dans beaucoup de pays européens, les salaires minimums représentent une fraction importante du salaire moyen. Associés à des coûts non salariaux importants, ils rendent souvent peu intéressant d'embaucher un travailleur non qualifié ou peu qualifié. Ainsi, les travailleurs peu qualifiés restent au chômage, et perdent par la même occasion l'opportunité d'acquérir une formation sur le tas ».

Cette hypothèse est-elle validée par les faits? Le tableau suivant indique les changements des salaires relatifs des travailleurs non qualifiés par rapport aux travailleurs qualifiés, et les taux de chômage relatifs, de la fin des années 1970 au début des années 1990, pour les États-Unis et quatre pays européens.

Tableau 5: Changement dans les salaires et chômage relatifs des travailleurs non qualifiés

\begin{tabular}{|c|c|c|c|}
\hline \multirow[t]{2}{*}{ Pays } & \multirow{2}{*}{$\begin{array}{c}\begin{array}{c}\text { Évolution } \\
\text { du salaire } \\
\text { relatif des }\end{array} \\
\begin{array}{c}\text { travailleurs } \\
\text { non qualifiés }\end{array} \\
\text { Entre } 1980 \\
\text { et } 1995(\%) \\
\end{array}$} & \multicolumn{2}{|c|}{$\begin{array}{l}\text { Taux de chômage relatif } \\
\text { des travailleurs non } \\
\text { qualifiés par rapport aux } \\
\text { travailleurs qualifiés }\end{array}$} \\
\hline & & $\begin{array}{c}\text { Fin des } \\
\text { années } 1970\end{array}$ & $\begin{array}{c}\text { Début des } \\
\text { années } 1990\end{array}$ \\
\hline États-Unis & -13 & 3,5 & 3,5 \\
\hline France & 3 & 1,5 & 2,9 \\
\hline Allemagne & 8 & 2,1 & 2,6 \\
\hline Italie & 1 & 0,4 & 1,1 \\
\hline Royaume-Uni & -14 & 3,1 & 5,3 \\
\hline
\end{tabular}

Source: emploi mondial 1996-1997, ILO, 1996.

Première colonne: évolution du salaire des travailleurs hommes du dernier décile de l'ensemble de la distribution des travailleurs, relativement au salaire des travailleurs du décile moyen. Deuxième et troisième colonnes : ratio du taux de chômage du dernier quartile de la population active, ordonnée par qualification (niveau d'étude), sur le taux de chômage du premier quartile.

On remarque que le salaire relatif des non-qualifiés a diminué aux États-Unis et au Royaume-Uni alors qu'il augmente dans les trois autres européens choisis. Le chômage relatif a augmenté de façon significative dans chacun des quatre pays européens, alors qu'il est resté à peu près constant aux États-Unis. Notons toutefois que le niveau de chômage relatif des non-qualifiés reste inférieur au niveau américain en France, Allemagne et Italie. Le seul pays où il lui est supérieur est le Royaume-Uni, où la baisse des salaires a pourtant été comparable à l'évolution américaine.

\section{Coûts de licenciements}

«Les entreprises européennes qui veulent licencier ont à supporter des coûts de licenciement importants, ou doivent s'engager dans des procédures judiciaires longues. Ces coûts non seulement empêchent de licencier des travailleurs qui ne sont pas nécessaires (ce qui augmente les coûts de production), mais ils poussent les entreprises à y réfléchir à deux fois avant d'embaucher un nouveau travailleur».
Une manière simple de mesurer l'influence de ces facteurs est de comparer les taux d'entrée et sortie dans le chômage de nos quatre pays.

Tableau 6: Entrées et sorties du chômage (2004)

\begin{tabular}{|l|r|r|r|r|}
\hline & Entrée & \multicolumn{1}{|c|}{ Sortie } & $\begin{array}{r}\text { Chômage de } \\
\text { longue durée }\end{array}$ & $\begin{array}{c}\text { Ratio E/ } \\
(\mathbf{E}+\mathbf{S})\end{array}$ \\
\hline USA & 2,02 & 33,0 & 12,7 & $5,8 \%$ \\
\hline France & 0,45 & 4,1 & 41,6 & $9,8 \%$ \\
\hline Danemark & 1,01 & 19,2 & 22,6 & $5,0 \%$ \\
\hline Pays-Bas & 0,32 & 4,6 & 32,5 & $6,4 \%$ \\
\hline
\end{tabular}

Source: (séries emploi, chômage, chômeurs depuis moins d'un mois, chômeurs depuis plus d'un an) données disponibles sur le site stats.oecd.org/wbos

Note: entrée et sortie: flux annuels d'entrants et de sortants du chômage rapportés aux populations d'origine (emploi total/ chômage). Entrants : chômeurs depuis moins d'un mois. Sortants : entrants moins variation du stock de chômeurs. Chômage de longue durée: part des chômeurs depuis douze mois ou plus dans le chômage total (en pourcentage).

La comparaison entre la France et les États-Unis est bien connue: la probabilité de passer par le chômage et d'en sortir est très supérieure aux ÉtatsUnis. La comparaison entre le Danemark et les Pays-Bas est également très parlante. Le Danemark est le pays européen où le passage par le chômage est le plus élevé (devant le Royaume-Uni). Il se situe à mi-chemin entre les États-Unis et l'Europe. Les Pays-Bas sont à l'inverse le pays où le risque d'entrer au chômage est le plus bas, en deçà du niveau français. Étant légèrement meilleur que les Français en matière de sortie du chômage, ils enregistrent au bout du compte des résultats comparables aux ÉtatsUnis (pour le ratio des deux). On retrouve dans ces exemples un résultat également connu: il n'existe pas de lien fiable entre «flexibilité» et chômage.

La conclusion s'impose : il n'existe pas une voie unique vers le plein-emploi. Telle est la principale innovation de la stratégie révisée pour l'emploi de l'OCDE (2006), laquelle souligne ainsi qu'il existe plusieurs combinaisons institutionnelles permettant de réduire le chômage. La diversité des trajectoires ne signifie évidemment pas qu'on puisse faire n'importe quoi. Mais elle oblige à mettre en vis-à-vis les différentes expériences et leurs exigences. On peut payer des indemnités élevées, mais à condition de disposer d'un dispositif de monitoring du retour à l'emploi efficace (Danemark). On peut atteindre le plein-emploi avec un turnover faible, mais à condition de gérer la formation des salaires de manière appropriée (Pays-Bas).

\section{La structure par âge}

Un axe d'analyse longtemps négligé mais pourtant essentiel tient à l'analyse par âge du marché de l'emploi. Cet aspect est riche d'enseignements sur les causes extérieures aux institutions du marché du travail qui gouvernent le sous-emploi. 
Tableaux 7 : Taux d'emploi par âge

\begin{tabular}{|l|r|r|r|r|}
\hline \multicolumn{1}{|c|}{ Âge } & \multicolumn{15}{c|}{$\mathbf{1 5 4}$} & $\mathbf{1 5 - 2 4}$ & $\mathbf{2 5 - 5 4}$ & $\mathbf{5 5 - 6 4}$ \\
\hline France & 62,3 & 26 & 79,6 & 40,7 \\
\hline États-Unis & 71,5 & 53,9 & 79,3 & 60,8 \\
\hline
\end{tabular}

Considérons tout d'abord le taux d'emploi des 25-54 ans. Il est équivalent en France et aux ÉtatsUnis (tant pour les hommes que pour les femmes). Si le contrat de travail français était véritablement inefficient, provoquant une attrition des postulants au marché du travail, il devrait se traduire par un moindre emploi de la catégorie centrale, celle des 25-54 ans, qu'il s'agisse des hommes ou des femmes. Dans le modèle canonique de Mortensen et Pissarides, par exemple, les frictions diverses introduites par un marché du travail inefficace déprime l'emploi, toutes catégories de travailleurs confondus. Le fait que le cœur des personnes âgées de 25 à 54 ans travaille autant aux Etats-Unis qu'en France pose une question spécifique à ces modèles.

Un article récent de BERTOLA et al. (2006) analyse cette question de manière approfondie. Le cœur de leur argument est le suivant. Le chômage est plus fort pour les catégories dont le coût d'opportunité $\mathrm{du}$ non-emploi est le plus faible. L'allongement de la durée des études et le raccourcissement de l'âge de la retraite en France expliquent, selon ce schéma, la montée du sous-emploi de ces catégories. Examinons ces deux catégories.

Commençons par le chômage des jeunes. Le CDD joue certainement un rôle important dans le chômage des jeunes, dans la mesure où il les oblige à un turnover artificiellement élevé. Mais d'autres facteurs doivent être pris en compte. Quand on regarde l'emploi des jeunes américains, le nombre d'heures travaillées est très faible, car il s'agit de jeunes travaillant bien souvent pour payer leurs études. Ce qui n'est pas un mal en soi, mais peut expliquer une partie de la différence avec la France où les études coûtent beaucoup moins cher.

Un fait paradoxal a été souligné par Dominique Goux et Cyril Nouveau (2007): le taux de chômage des jeunes est resté stable, alors même que l'allongement de la durée des études a réduit leur expérience professionnelle. Lorsqu'on décale la fenêtre d'observation du chômage «jeune» d'une durée qui correspond à l'accroissement de la durée des études, on trouve un taux d'emploi des «jeunes» de 27-32 ans qui monte au cours du temps. Davantage que le contrat de travail, il est donc possible d'incriminer d'autres facteurs, tel l'allongement de la durée d'études dans le sous-emploi des jeunes français.

Le chômage des seniors est l'autre cause du sous-emploi. C'est le point où la France marque un déséquilibre patent avec les États-Unis. Pour les 55-64 ans, le taux d'emploi s'élève à $47 \%$ pour les hommes, contre 70\% aux États-Unis. Pour les femmes, le chiffre est de $40 \%$ en France, contre $57 \%$ aux États-Unis. Cet énorme dégradé ne peut pas être analysé indépendamment d'un facteur majeur: l'abaissement de l'âge de la retraite en France. Les études montrent très clairement que le chômage des seniors en France est principalement expliqué par ce facteur, comparativement à son niveau il y a trente ans ou à d'autres pays. Ce n'est pas l'âge biologique des personnes qui explique leur sousemploi, mais leur âge social, à savoir le temps qu'il leur reste à travailler avant de prendre leur retraite (cf. le rapport du Conseil d'analyse économique d'Antoine D'Autume et Jean-Olivier Hairault, 2006). On est ici dans un domaine qui ne relève pas directement des questions classiques du marché du travail, où le contrat de travail et la politique macroéconomique joueraient le rôle dominant. La question centrale, simple et mécanique est de savoir quel est l'horizon temporel des travailleurs, quel est l'horizon sur lequel l'investissement que représente une embauche ou la recherche d'un emploi peut être recouvré.

\section{La productivité}

Un aspect important des critiques apportées aux institutions d'Europe continentale est d'empêcher l'allocation optimale du travail. La faible mobilité des travailleurs, selon cet argument, agirait comme un frein à l'appariement (le matching) efficace des travailleurs et des firmes. Qu'en est-il au juste?

La figure et le tableau suivants sont tirés des travaux de VAN ARK et PiatKowsKi (2004) du centre Groningen pour l'emploi. La figure 1 montre l'évolution de la productivité française, analysée relativement à la productivité américaine. On voit que la convergence du PIB par habitant français vers le niveau américain s'interrompt au début des années 1980, tandis que la productivité horaire surajuste le niveau américain, avant de se stabiliser puis de décliner légèrement à partir de 1995. Le tableau 2 donne la comparaison des variables qui permettent de passer de la productivité horaire au revenu par habitant dans nos quatre pays. 
Figure 1 :

PIB, РIB par habitant et PIB par heure travaillèe, 1959-2006

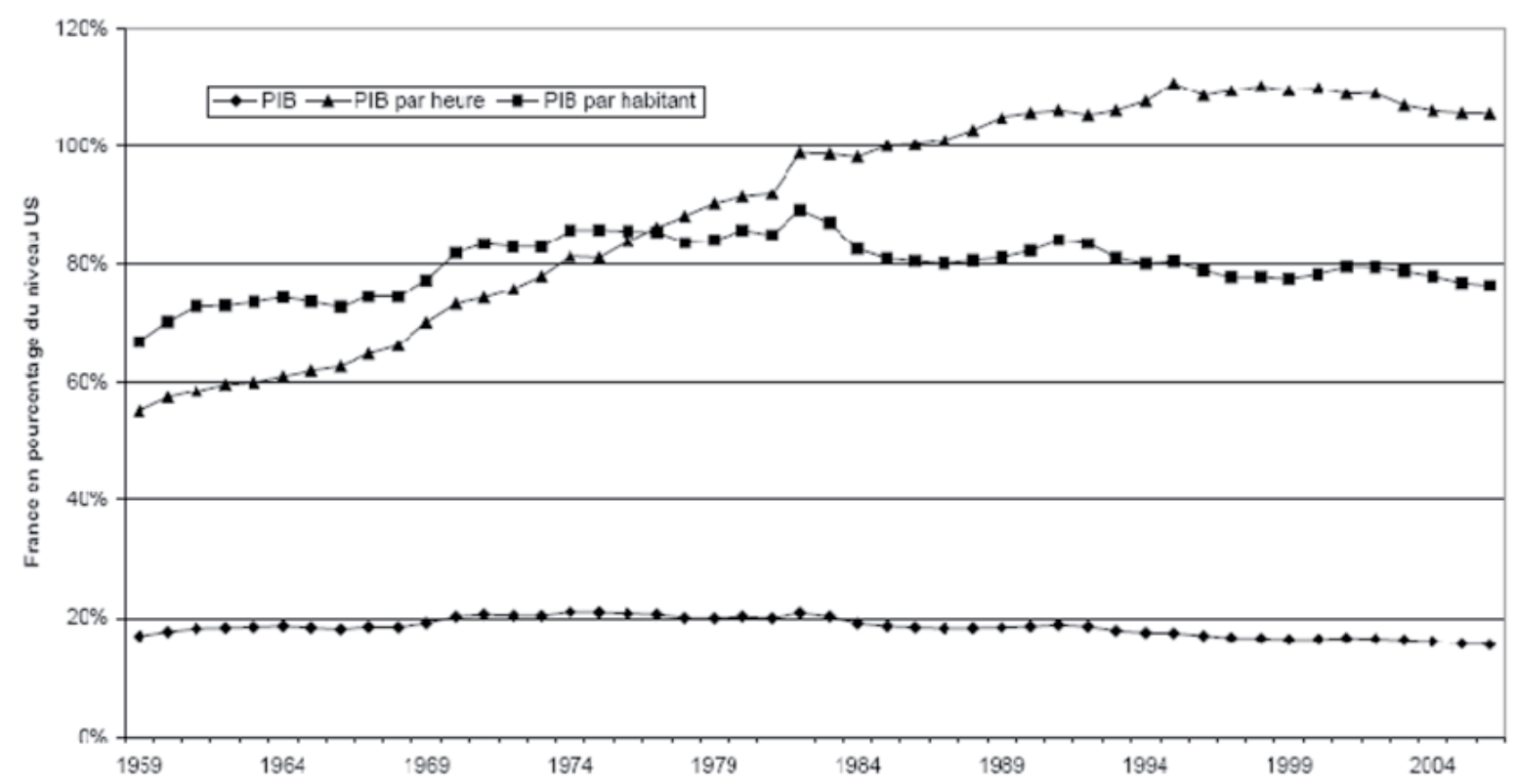

Source: $\mathrm{h}$ ttp://www.ggdc.net/dseries/totecom.html

Table 8 :Labour Productivity and Income : Differences in Ranking, 2004

\begin{tabular}{|l|c|c|c|c|c|c|c|c|}
\hline & \multicolumn{2}{|l|}{ Productivity (per hour) } & \multirow{2}{*}{$\begin{array}{c}\text { Effect of } \\
\text { Working Hours }\end{array}$} & $\begin{array}{c}\text { Productivity (per } \\
\text { worker) }\end{array}$ & $\begin{array}{c}\text { Effects of } \\
\text { Employment/ } \\
\text { Pop.Ratio }\end{array}$ & \multicolumn{2}{|l|}{ Per Capita Income } \\
\cline { 5 - 8 } & US\$ & \%US & & US\$ & $\%$ US & & \\
\hline EU-15a & 40,51 & $91 \%$ & $-13 \%$ & 63311 & $78 \%$ & $-6 \%$ & 27666 & $72 \%$ \\
\hline Luxembourg & 56,84 & $128 \%$ & $-24 \%$ & 83959 & $104 \%$ & $37 \%$ & 53993 & $141 \%$ \\
\hline France & 50,08 & $113 \%$ & $-24 \%$ & 72065 & $89 \%$ & $-14 \%$ & 28956 & $76 \%$ \\
\hline Belgium & 48,12 & $109 \%$ & $-13 \%$ & 76890 & $95 \%$ & $-18 \%$ & 29826 & $78 \%$ \\
\hline Ireland & 46,26 & $104 \%$ & $-10 \%$ & 76274 & $95 \%$ & $-3 \%$ & 35021 & $91 \%$ \\
\hline Netherlands & 44,48 & $100 \%$ & $-26 \%$ & 60278 & $75 \%$ & $3 \%$ & 29766 & $78 \%$ \\
\hline Austria & 43,81 & $99 \%$ & $-17 \%$ & 65646 & $81 \%$ & $-2 \%$ & 30466 & $79 \%$ \\
\hline Germany & 43,22 & $97 \%$ & $-20 \%$ & 62349 & $77 \%$ & $-7 \%$ & 27076 & $71 \%$ \\
\hline Denmark & 41,65 & $94 \%$ & $-17 \%$ & 62364 & $77 \%$ & $3 \%$ & 30746 & $80 \%$ \\
\hline Finland & 39,60 & $89 \%$ & $-8 \%$ & 65414 & $81 \%$ & $-4 \%$ & 29545 & $77 \%$ \\
\hline U.K. & 39,28 & $89 \%$ & $-10 \%$ & 63676 & $79 \%$ & $-1 \%$ & 29935 & $78 \%$ \\
\hline Italy & 39,27 & $89 \%$ & $-11 \%$ & 62930 & $78 \%$ & $-8 \%$ & 26714 & $70 \%$ \\
\hline Sweden & 39,24 & $88 \%$ & $-12 \%$ & 61789 & $77 \%$ & $0 \%$ & 29517 & $77 \%$ \\
\hline Spain & 32,59 & $73 \%$ & $-1 \%$ & 58583 & $73 \%$ & $-8 \%$ & 24763 & $65 \%$ \\
\hline Greece & 28,14 & $63 \%$ & $3 \%$ & 53978 & $67 \%$ & $-11 \%$ & 21326 & $56 \%$ \\
\hline Portugal & 22,53 & $51 \%$ & $-3 \%$ & 38715 & $48 \%$ & $1 \%$ & 18909 & $49 \%$ \\
\hline
\end{tabular}

Source: VAN ARK (2005).

La comparaison donnée entre la France et les États-Unis est fascinante. En productivité horaire, la France est $13 \%$ plus productive que les ÉtatsUnis. Ce chiffre tient évidemment en partie à l'attrition des travailleurs les moins productifs. Gilbert Cette a proposé une correction pour ce terme, dont il ressort que la France est structurellement moins productive que les États-Unis, de $8 \%$ environ.
La différence entre cette productivité horaire et le revenu par habitant tient mécaniquement à deux facteurs: le nombre d'heures travaillées par travailleur, et le nombre de travailleurs employés en pourcentage de la population. Relativement aux États-Unis, les travailleurs français travaillent 24\% de moins que les Américains. Le tableau suivant montre l'évolution des heures travaillées en France 
et aux États-Unis, analysée comparativement aux données suédoises.

Tableau 9 : Nombre d'heures annuelles par travailleur

\begin{tabular}{|l|r|r|}
\hline & $\mathbf{1 9 7 9}$ & $\mathbf{2 0 0 5}$ \\
\hline France & 1856 & 1535 \\
\hline États-Unis & 1834 & 1804 \\
\hline Suède & 1530 & 1587 \\
\hline
\end{tabular}

On voit que la France travaillait comme les ÉtatsUnis en 1979, et qu'elle a rejoint le nombre d'heures suédois en fin de période. Cette question ouvre au débat controversé sur la préférence française pour le loisir selon Blanchard, ou des effets pervers de la fiscalité selon Prescott, que nous ne trancherons pas ici.

Le tableau donne enfin l'effet du moindre taux d'emploi entre les deux pays. La France enregistre un taux d'emploi qui est $14 \%$ inférieur au niveau américain. On voit que son effet est presque deux fois moins important que le nombre d'heures travaillées dans l'explication du revenu par habitant.

Au total, ces chiffres rendent difficile de conclure que le moindre turnover français ou hollandais handicape significativement la productivité de ces pays par rapport à ceux où il est plus fort, comme le Danemark ou le Royaume-Uni. Ce dernier pays est en fait celui où la productivité horaire est la plus faible du groupe.

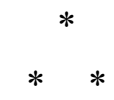

Au terme de ce tour d'horizon, il est possible de conclure que le sous-emploi français ne doit pas principalement aux institutions du marché du travail (syndicats, indemnités, coûts de licenciements) mais à d'autres facteurs qui lui sont en partie extérieurs: la gestion défectueuse de la fin d'études ou l'âge de la retraite précoce. Un point essentiel doit sans doute être aussi pris en compte. La différence de niveau d'études entre les plus de 55 ans et les moins de 30 ans est considérable en France, du fait de l'allongement brutal des années d'études dans les années 1980. Les déficiences notoires du système de formation professionnelle française jouent un rôle difficile à tester mais certainement crucial, dans la décision de licencier des plus de 50 ans, alors même qu'ils sont souvent, par hypothèse, ceux dont les coûts de séparation sont les plus chers.

\section{Bibliographie}

Algan Y., Cahuc P. (2005), "The Roots of Low European Employment: Family Culture?", NBER Macroeconomic Annuals, MIT Press.

Algan Y., Cahuc P. (2006), "Civic Attitudes and the Design of Labor Markets Institutions", Mimeo, CREST.

BEAN C. (1994), "European unemployment: a survey", Journal of Economic Literature.

Blanchard O. (2004), “The Economic Future of Europe”, Journal of Economic Perspectives.

Blanchard O., Philippon T. (2005), The quality of Labor Relations and Unemployment, NBER, Working Paper 10590.

Blanchard O., Cohen D. (2007), Macroéconomie, Pearson Education.

D’Autume A., Betbeze J.-P., Hairault J.-O. (2005), Les seniors et l'emploi en France, 2005, rapport du Conseil d'analyse économique, Paris, La Documentation française.
Goux D., Nouveau C. (2006), «Expansion scolaire et insertion professionnelle: une évaluation de l'ouverture du lycée depuis le début des années 1980» in Une Jeunesse Difficile. Portrait économique et social de la jeunesse française CEPREMAP, éditions ENS Rue d'Ulm.

Giuseppe B., Blau F., Kahn L. (2006), "Labor Market Institutions and Demographic Employment Patterns" Journal of Population Economics.

LaRs C., Driffill J. (1989), "Bargaining structure, corporatism, and macroeconomic performance", Economic Policy, № 6, 1988.

OCDE (2006), Perspectives de l'emploi de l'OCDE. Stimuler l'emploi et les revenus, disponible sur le site: http:// www.sourceocde.org/emploi/9264023860

Van Ark B., Piatkowski M. (2004), "Productivity, innovation and ICT in Old and New Europe", International Economics and Economic Policy, Vol. 1, pp. 215-246. 